\title{
Analysis of Sum Rules \\ Following from Local Commutation Relations of Currents.
}

\author{
F. Buccella and G. Veneziano \\ Istituto di Fisica dell' Università - Firenze. \\ R. Gatto \\ Istituto di Fisica dell'Universitì - Firenze \\ Istituto Nazionale di Fisica Nucleare - Sezione di Firená \\ (Nuovo Cimento, 42 A, $1019(1966)$ )
}

p. 1019, first formula, instead of

read

$$
\frac{1}{3}\left[\left\langle r_{\mathrm{p}}^{2}\right\rangle-\left\langle r_{\mathrm{n}}^{2}\right\rangle\right]=\left(\frac{\mu_{\mathrm{p}}-\mu_{\mathrm{n}}}{2 m}\right)^{2}\left(\frac{g_{A}}{g_{v}}\right)^{2}
$$

$$
\frac{1}{3}\left[\left\langle r_{\mathrm{p}}^{2}\right\rangle-\left\langle r_{\mathrm{n}}^{2}\right\rangle\right]=\left(\frac{\mu_{\mathrm{p}}-\mu_{\mathrm{n}}}{2 m}\right)^{2}\left(\frac{g_{v}}{g_{A}}\right)^{2},
$$

p. 1020, eq. (3), instead of

$$
\ldots 2 K_{0} K_{0}^{\prime} \cos ^{2} \frac{\Phi}{0}
$$

read

$$
\ldots 2 K_{0} K_{0}^{\prime} \cos ^{2} \frac{\Phi}{2}
$$

p. 1026, eq. (36), instead of

$$
\left(\frac{\mu_{\mathrm{p}}-\mu_{\mathrm{n}}}{2 m}\right)^{2}=\frac{25}{9} \frac{\mu_{\mathrm{p}}^{\mathrm{n}}}{4 m^{2}}
$$

rearl

$$
\left(\frac{\mu_{\mathbf{p}}-\mu_{\mathrm{u}}}{2 m}\right)^{2}=\frac{25}{9} \frac{\mu_{\mathrm{p}}^{2}}{4 m^{2}},
$$

p. 1019 , ref. $\left({ }^{3}\right)$, instead of

... Phys. Lett., 19, 697 (1965).

read

... Phys. Lett., 19, 697 (1966).

PROPRIETA LETTERARIA RISERVATA

Direttore responsabile: G. BERNARDINI

Tipografia Compositori - Bologna 